Short Report

\title{
Molecular Identification of a Clinical Isolate of Non- pigmented and Asporogenic Aspergillus fumigatus Strain Which cannot be Identified by Traditional Morphological Methods
}

\author{
Kayo Watanabe, Masakazu Katsu, Miki Tamura, Katsukiyo Yazawa, \\ Katsuhiko Kamei, Yuzuru Mikami, Kazuko Nishimura \\ Research Center for Pathogenic Fungi and Microbial Toxicoses, Chiba University, \\ 1-8-1 Inohana Chuo-ku, Chiba 260-8673, Japan
}

[Received: 14, November 2000. Accepted: 26, December, 2000]

\begin{abstract}
A non-pigmented and asporogenic fungal strain without taxonomically useful characteristics was isolated from sputum of a patient with chronic bronchitis. Although the taxonomic position could not be determined based on traditional morphological criteria, the fungus was identified as an Aspergillus fumigatus mutant strain by molecular techniques including ITS sequence analysis and PCR identification system using a PCR primer pair specific for A. fumigatus. Usefulness of these molecular techniques for the identification of non-classifiable fungal strains in a clinical laboratory is discussed.

Key words: ITS sequence, RAPD pattern, clinical specimen, Aspergillus
\end{abstract}

A non-pigmented and asporogenic fungal strain (260A) without taxonomically useful morphological characteristics was isolated from the sputum of a 70 year old male with chronic bronchitis in Chiba University Hospital. Color of the fungal colonies of the $260 \mathrm{~A}$ strain was at first white, and then the margins changed to grayish white with a greenish tinge. Since taxonomically useful morphological characters were not observed, molecular techniques were applied to identify this organism. Internal transcribed spacer (ITS) and 5.8S rDNA region nucleotide sequences (ITS15.8S rDNA-ITS2 region) have been considered one of the most suitable molecular techniques for examining species level variability for pathogenic fungi ${ }^{1)}$. Other useful molecular techniques employed for the identification were PCR amplification using specific primers for $18 \mathrm{~S}$ - or $28 \mathrm{~S}$ rRNA gene sequences and RAPD band pattern analysis.

In this manuscript, the results of this approach are reported and usefulness of other molecular

Correspondince: Dr. Yuzuru Mikami

Research Center for Pathogenic Fungi and Microbial

Toxicoses, Chiba University,

1-8-1 Inohana, Chuo-ku, Chiba 260-8673, Japan. methods such as RAPD band pattern analysis is also discussed.

Strain 260A was isolated from the patient (Dec., 1999), and inoculated in a potato dextrose agar (PDA) slant. Slide culture of the fungus showed production of conidiophores with conidiogenous cells which were related to the morphological structures of Aspergillus species, but no morphological features distinctive enough for the species identification were observed (Fig. 1). Production of conidiospores was very rare, and at most one conidium per conidiogenous cell. Therefore, molecular methods were used to identify this fungus and DNA of the strain was extracted by modified methods of Aoki et al. ${ }^{2)}$ and Imai et $a l .^{3,4)}$. Briefly, two or three blocks of the fungal debris from the agar slants were suspended in $200 \mu l$ of TE buffer (10 mM Tris$\mathrm{HCl}, \mathrm{pH}$ 8.0, $1 \mathrm{mM}$ EDTA) in an Eppendorf tube. Then, the DNA was extracted with GPT reagent (6 M guanidine thiocyanate dissolved in $50 \mathrm{mM}$ Tris [pH 8.3]) and phenol buffered in Tris ( $\mathrm{pH}$ 8.0). After extraction with chloroformisoamyl alcohol solution, the aqueous phase was separated by centrifugation, and mixed with an equal amount of isopropanol with $3 \mathrm{M}$ sodium 


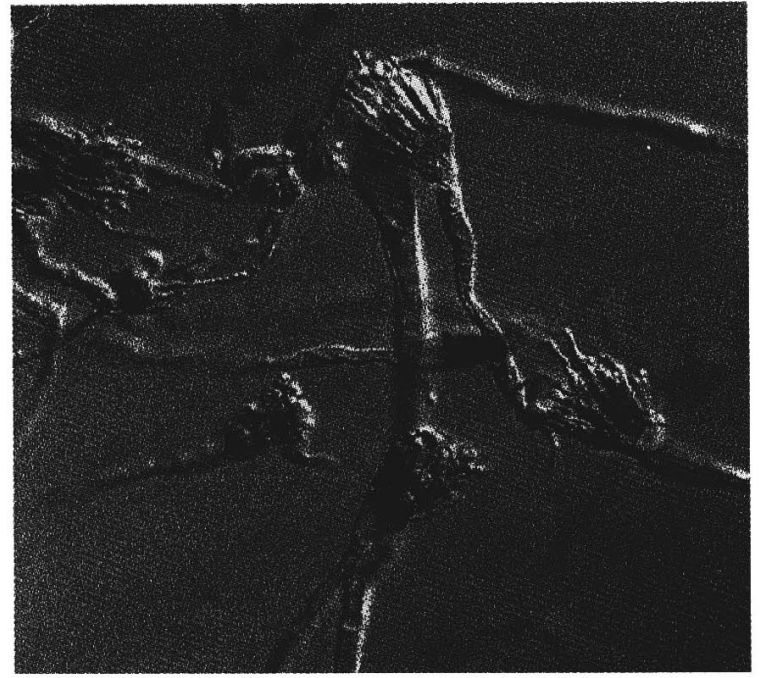

Fig. 1. Morphology of non-pigmented and asporogenic $A$. fumigatus $260 \mathrm{~A}$ strain.

acetate. Samples were centrifuged, and the nucleic acid pellet was washed with $70 \%$ ethanol, dried, and resuspended in sterile TE-buffer at a DNA concentration of $5 \mu \mathrm{g} / \mathrm{m} l$. All reaction products were observed by electrophoresis on $1.5 \%$ agarose gels in $1 \times$ TBE buffer at $70 \mathrm{~V}$ for $100 \mathrm{~min}$ and then stained in $0.5 \mu \mathrm{g} / \mathrm{ml}$ of ethidium bromide (EB) solution. For determination of ITS region DNA sequence, PCR primers of ITS $5=5$-GGAAGTAAAAGTCGTAACAAGG3' and ITS4 $=5$ '-TCCTCCGCTTATTGATATGC3' were used ${ }^{1)}$. Amplification reactions were performed in a final volume of $32.5 \mu l$ of distilled water containing $2.5 \mu l$ of each primer $(20 \mathrm{pM})$, $2.5 \mu l$ of genomic DNA $(5 \mu \mathrm{g} / \mathrm{ml})$ and one PCR bead (Ready-To-Go ${ }^{\mathrm{TM}}$ PCR beads, Pharmacia Biotech), and the amplification conditions were the same. The PCR products were sequenced using an ABI Prism BigDye Terminator Cycle Sequencing Ready Reaction Kit (Applied Biosystems). Analyses of DNA sequence reactions were aligned using CLUSTAL $\mathrm{W}$ version $1.74^{5)}$ and manually adjusted.

The determined ITS region sequence of the 260A strain was $538 \mathrm{bp}$ (deposited to DDBJ as accession number AB051071), and was compared with those of reference $A$. fumigatus strain (accession number AF07888). The sequence similarity of $99.44 \%$ suggested that strain $260 \mathrm{~A}$ was $A$. fumigatus. The phylogenetic tree prepared based on the ITS sequence information confirmed this identification, despite the absence of morphological characteristics of $A$. fumigatus species (data not shown). These data supported our hypothesis that this strain is an asporogenic mutant of $A$. fumigatus ${ }^{6)}$.

PCR primer pairs designed to amplify the 401

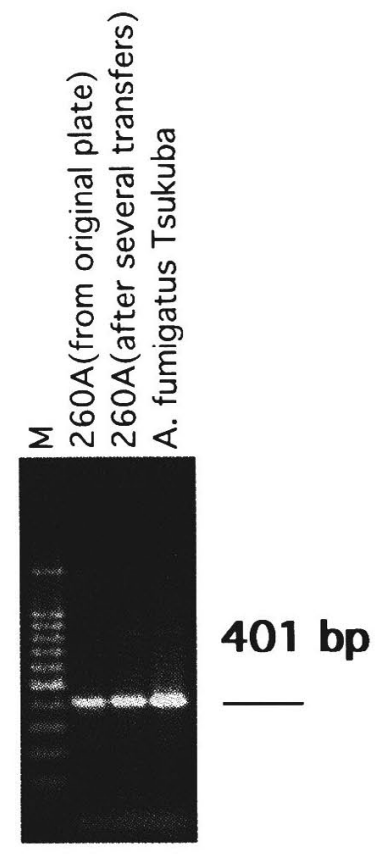

Fig. 2. PCR identification of non-pigmented and asporogenic A. fumigatus $260 \mathrm{~A}$ strain by PCR. PCR primer pair was used to amplify a $401 \mathrm{bp}$ fragment spanning the $26 \mathrm{~S} /$ intergenic spacer region of the rDNA complex of $A$. fumigatus.

A ladder was used as the molecular size standard (M), and each band shows $100 \mathrm{bp}$ size difference. $\mathrm{R}$ shows $A$. fumigatus Tsukuba reference strain.

bp band of $A$. fumigatus based on the 26S/IGS region sequence information have been proposed ${ }^{7)}$. Testing these primers for the identification, we learned that the primer sequences were incorrect so we modified the antisense-primer sequences based on the rDNA sequence information. Since we have confirmed the usefulness of the modified PCR primer pairs for the identification of $A$. fumigatus in many clinical isolates (unpublished data), we tested the DNA from 260A. The amplified $401 \mathrm{bp}$ band from this DNA sample is shown in Fig. 2. The result also supported our identification of it as $A$. fumigatus species.

During several transfers of $A$. fumigatus $260 \mathrm{~A}$ in PDA slants, one culture which showed greenish colonies was obtained. Observation of the greenish strain suggested that it was a revertant strain of A. fumigatus 260A (designated as 260A$\mathrm{M})$, showing similar morphological characteristics to the reference $A$. fumigatus. Since RAPD analysis method has been applied to detect variability in strain level of fungi, we were interested in the RAPD pattern of the revertant (260A-M strain). Three primers were used for the RAPD analysis: R-1 = 5'-ATGGA TCGGC-3', R-2 = 5'-A TTGCGT CCA-3' and R-3=5'-TCACGATGCA-3', and 


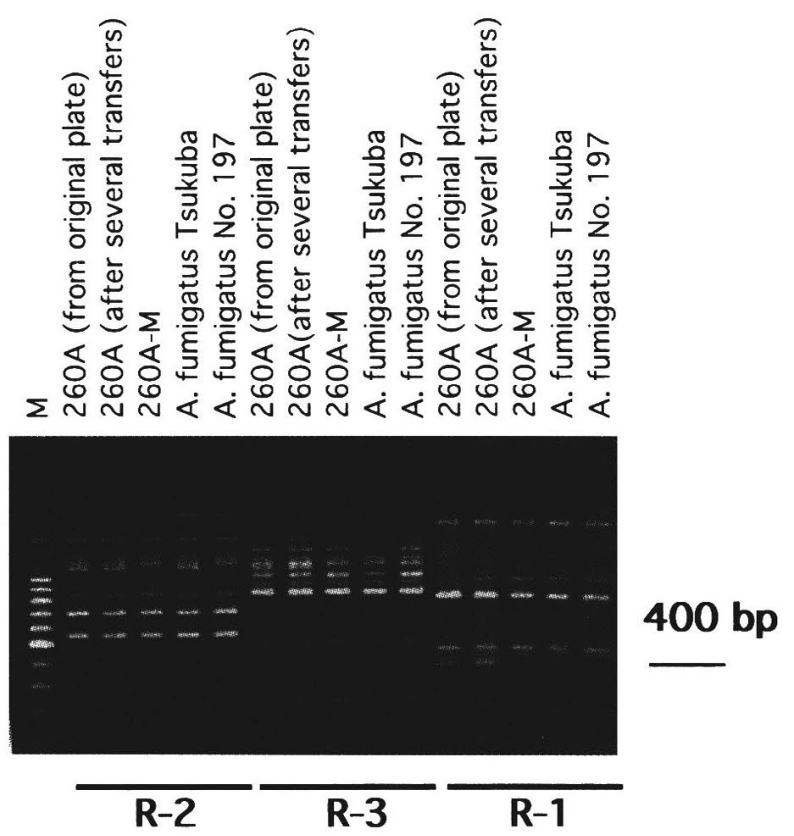

Fig. 3. RAPD fingerprint band patterns of $A$. fumigatus $260 \mathrm{~A}$ and its revertant strain in comparison with those of reference strains of $A$. fumigatus.

R-1 primer, R-2 and R-3 are the RAPD primers used in this experiment.

A. fumigatus Tsukuba and No.197 strains were used as the references.

amplification reactions were performed by the same methods reported in our previous paper ${ }^{8)}$.

RAPD band patterns of $A$. fumigatus 260A were compared with those of the reference $A$. fumigatus strains (Tsukuba and No. 197 strains), and the results are shown in Fig. 3. No RAPD band pattern difference was observed whether the DNA samples were obtained from the original plate or after several transferred plates. $A$. fumigatus 260A showed characteristic RAPD band patterns with the three primers tested, and the amplified band sizes and patterns were identical with those of the reference $A$. fumigatus strains with R-2 and R-3 primers. Interestingly, when we used R-1 primer, although other amplified RAPD band patterns were identical, an additional $400 \mathrm{bp}$ band was observed in the DNA sample of $A$. fumigatus 260A. The RAPD pattern of the possible revertant strain (260A-M) was almost identical with those of $A$. fumigatus reference strains as well as the 260A strain. Further detailed genetic analysis is now in progress in our laboratory to confirm its fungal origin. This RAPD pattern analysis suggested that this method is also useful for the identification of $A$. fumigatus strains if appropriate reference strains are used. To our knowledge, this is the first report of the isolation of an asporogenic $A$. fumigatus strain from a clinical sample.

\section{References}

1) White TJ, Bruns $T$, Lee $S$, Taylor J: Amplification and direct sequencing of fungal ribosomal RNA genes for phylogenetics. In "PCR Protocols: A Guide to Methods and Applications" Academic Press, San Diego 1990, pp.315-322.

2) Aoki FH, Imai T, Tanaka R, Mikami Y, Taguchi H, Nishimura NF, Nishimura K, Miyaji M, Schreiber AZ, Branchini MLM: New PCR primer pairs specific for Cryptococcus neoformans serotype A or B prepared on the basis of random amplified polymorphic DNA fingerprint pattern analyses. J Clin Microbiol 37: 315320, 1999.

3) Imai $T$, Sano A, Mikami Y, Watanabe K, Aoki FH, Branchini MLM, Negroni R, Nishimura NF, Nishimura K, Miyaji M: A new PCR primer for the identification of Paracoccidioides brasiliensis based on rRNA sequences coding the internal transcribed spacers (ITS) and 5.8S regions. Medical Mycology 38: 323-326, 2000.

4) Imai T, Watanabe K, Tamura M, Mikami Y, Tanaka R, Nishimura K, Miyaji M, Poonwan N, Branchini MLM: Geographic grouping of Cryptococcus neoformans var. gattii by random amplified polymorphic DNA fingerprint patterns and ITS sequence divergence. Clinical Laboratory 46: 345-354, 2000.

5) Thomson JD, Higgins DG, Gibbson TD, Clustal W: Improving the sensitivity of progress of multiple sequence alignment through sequence weighting, position specific gap penalties, and weight matrix choice. Nucleic Acids Res 22: 2673-2680, 1994.

6) Levadoux W, Taylor A, Gregory KF: Sequential cold-sensitive mutations in Aspergillus fumigatus. II. Analysis of the parasexual cycle. Can J Microbiol 27: 295-303, 1981.

7) Spreadbury C, Holden D, Auauvre-brown A, Bainridge B, Cohen J: Detection of Aspergillus fumigatus by polymerase chain reaction. J Clin Microbiol 31: 615-621, 1993.

8) Poonwan N, Imai $T$, Mekha $N$, Yazawa $K$, Mikami Y, Ando A, Nagata Y: Genetic analysis of Histoplasma capsulatum strains isolated from clinical specimens in Thailand by a PCR-based random amplified polymorphic DNA method. J Clin Microbiol 36: 3073-3076, 1998. 